\title{
GPR56 homozygous nonsense mutation p.R271* associated with phenotypic variability in bilateral frontoparietal polymicrogyria
}

Tülay Öncü-Öner ${ }^{1}$, Aycan Ünalp², İlknur Porsuk-Doru ${ }^{1}$, Sinem Ağ1lkaya ${ }^{1}$, Handan Güleryüz ${ }^{3}$, Aydan Saraç ${ }^{4}$, Bekir Ergüner ${ }^{5}$, Bayram Yüksel ${ }^{4}$, Semra Hız-Kurul' Sultan Cingöz ${ }^{1}$

Departments of ${ }^{1}$ Medical Biology and Genetics, ${ }^{3}$ Pediatric Radiology, ${ }^{6}$ Pediatric Neurology, Dokuz Eylül University Faculty of Medicine; ${ }^{2}$ Department of Pediatric Neurology, Dr. Behcet Uz Children's Hospital, İzmir; ${ }^{4}$ Genetic Engineering and Biotechnology Institute, TUBITAK Marmara Research Center, ${ }^{5}$ Advanced Genomics and Bioinformatics Research Center (IGBAM), The Scientific and Technological Research Council of Turkey (TUBITAK), BILLGEM, Kocaeli, Turkey.

E-mail: sultan.cingoz@deu.edu.tr

Received: 20th July 2017, Revised: 8th September 2017, Accepted: 30th November 2017

SUMMARY: Öncü-Öner T, Ünalp A, Porsuk-Doru İ, Ağılkaya S, Güleryüz H, Saraç A, Ergüner B, Yüksel B, Hız-Kurul S, Cingöz S. GPR56 homozygous nonsense mutation p.R271* associated with phenotypic variability in bilateral frontoparietal polymicrogyria. Turk J Pediatr 2018; 60: 229-237.

Polymicrogyria is a disorder of neuronal migration characterized by excessive cortical folding and partially fused gyri separated by shallow sulci. Homozygous mutations in the GPR56 gene, which regulates migration of neural precursor cells, are associated with bilateral frontoparietal polymicrogyria (BFPP) syndrome including white matter changes, brainstem and cerebellar involvement. Herein, we describe three siblings of consanguineous parents with a homozygous germline mutation (p.R271*) located in the seventh exon of the GPR56 gene that was previously detected in only one Portuguese patient. Phenotypic/genotypic relationships were analysed according to the clinical characteristics in only index patient. While earlier reported patient was exhibiting seizures provoked by hot water, macrocephaly, cerebellar/brainstem hypoplasia and corpus callosum abnormalities, the index patient showed only hypoplasia of brainstem, focal onset bilateral tonic clonic seizure. Despite the phenotypic similarities in two patients, the potential causes of the variation in the expression of the p.R271* variant between the two affected families might be genetic or epigenetic factors beyond the GPR56 gene. Consequently, the present findings show that the same mutation in GPR56 gene can have different phenotypic effects. Therefore, additional functional studies are needed to detect the phenotypic spectrum of the p.R271* mutation in GPR56, and provide insight into the mechanism of normal cortical development and regional patterning of the cerebral cortex.

Key words: BFPP, GPR56 mutations, polymicrogyria, whole exome sequencing.

Polymicrogyria (PMG) is a cortical brain malformation characterized by excessive cortical folding and partially fused gyri separated by shallow sulci. ${ }^{1}$ In the past, the incidence of PMG was not precisely known because of limitations in imaging technology and was often misdiagnosed as pachygyria. Along with subsequent advancements in molecular genetics and MRI techniques the diagnosis and classification of PMG have improved and several region-specific PMG syndromes have been described. ${ }^{2}$ Bilateral PMG has been classified according to the radiological findings as frontal, frontoparietal, perisylvian, lateral parietal, parasagittal parieto-occipital and generalized. Radiological findings in patients with $\mathrm{G}$ protein-coupled receptor 56 (GPR56) gene mutations are characterized by 
frontoparietal distribution of PMG which is the most common feature of bilateral frontoparietal PMG (BFPP) syndrome. ${ }^{3,4}$ On the other hand, several reported patients with GPR56 mutations exhibit extensive distribution throughout the entire brain, including the occipital and temporal regions. ${ }^{5}$ Homozygous mutations in the GPR56 gene (OMIM \#604110), which regulates migration of neural precursor cells ${ }^{6}$, are associated with BFPP syndrome. All predicted pathogenic GPR56 variants, except homozygous deletion of one 15-bp tandem repeat in a regulatory region of the GPR56 gene in five patients with bilateral perisylvian PMG from three families were detected only in patients with typical BFPP syndrome (OMIM \#606854) which includes the white matter, brainstem and cerebellar changes. 5,7 BFPP syndrome without cerebellar and brainstem changes is referred to as BFPP2 syndrome, and has not been associated with any mutations in GPR56. ${ }^{4}$ Moreover, it was reported that patients

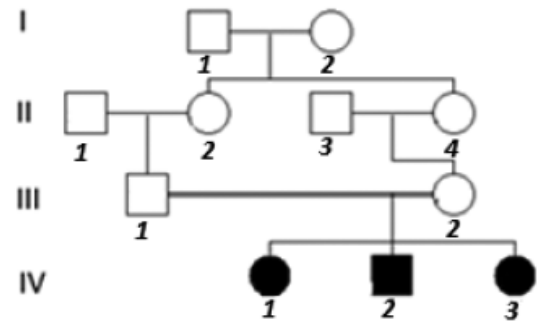

C

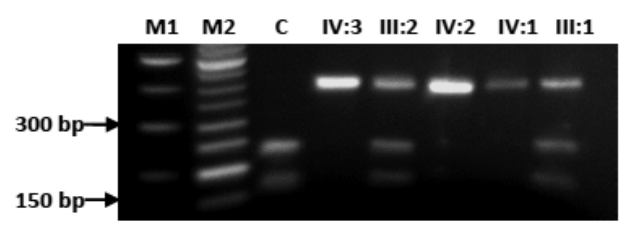

D

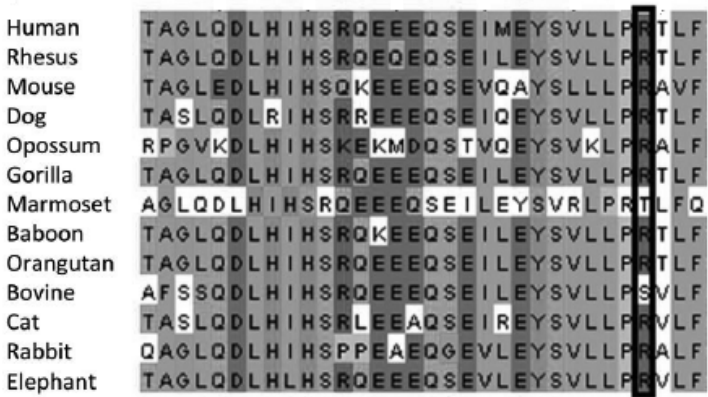

B

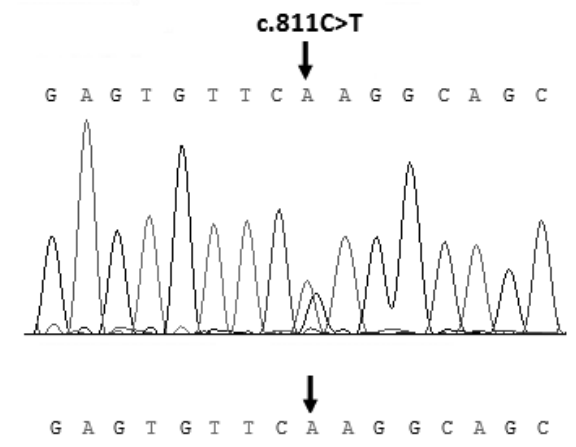

III:2

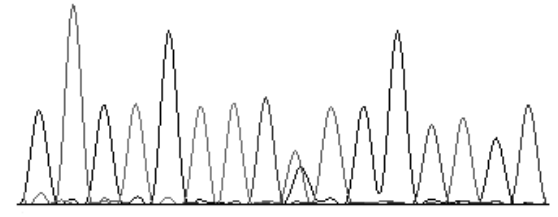

IV:1
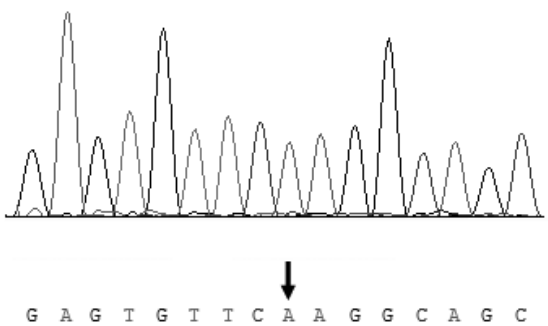

IV:2

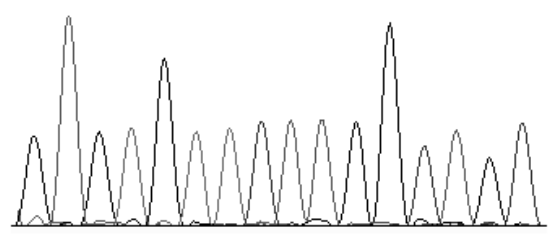

Fig 1. Detected c.811C>T (p.R271*) mutation in GPR56 gene A) The pedigree of the index family. Squares and circles indicate males and females, respectively. Solid symbols indicate affected individuals. B) Electropherograms of the partial sequence showing the mutation c.811C > T (p.R271*) in GPR56 in two siblings, Homozygous, and their parents III-1, III-2, Heterozygous. The arrow indicates the mutation. The quality of the Sanger chromatogram was poor for patient IV-3, so it wasn't shown. C) Agarose gel electrophoresis showing the confirmation and segregation of the mutation by Taq1 restriction enzyme cutting. M1:100bp DNA ladder, M2:50bp DNA ladder, C:Control, IV-1, IV-2, IV-3: patients, III-1, III-2: parents. D) Multiple sequence alignment of the region including the mutation (p.R271*) of human GPR56 gene across 13 species. The figure was generated using GRCh37/hg19, a ClustalW method, and Jalview Java alignment editor version 2 . 
Table I. Clinical Features of Patients with the p.R271* Mutation in GPR56 Gene.

\begin{tabular}{|c|c|c|}
\hline Clinical features & Our patients & $\begin{array}{l}\text { Patient reported by Santos-Silva et } \\
\text { al, } 19\end{array}$ \\
\hline Gender/age & $2 \mathrm{~F}, 1 \mathrm{M} / 14 \mathrm{y}, 23 \mathrm{y}$ and $25 \mathrm{y}$ & $\mathrm{M} / 5 \mathrm{y}$ \\
\hline Family history & $\begin{array}{l}\text { Consanguinity (3 siblings) } \\
\text { affected }\end{array}$ & No consanguinity \\
\hline Motor developmental delay & Severe & Severe \\
\hline Mental retardation & Severe & Severe \\
\hline Neurological examination & $\begin{array}{l}\text { Spastic diparesis, brisk deep- } \\
\text { tendon reflexes }\end{array}$ & $\begin{array}{l}\text { Axial hypotonia, flaccid } \\
\text { tetraparesis, brisk deep-tendon } \\
\text { reflexes in lower limbs }\end{array}$ \\
\hline Head size & Normal & Macrocephaly \\
\hline Type of the seizures & $\begin{array}{l}\text { Focal onset bilateral tonic clonic } \\
\text { seizure. }\end{array}$ & Seizures provoked by hot water \\
\hline EEG findings & $\begin{array}{l}\text { Epileptic discharges on } \\
\text { predominantly frontal areas }\end{array}$ & $\begin{array}{l}\text { Epileptic discharges on } \\
\text { predominantly frontal areas }\end{array}$ \\
\hline PMG distribution & Bilateral frontoparietal-occipital & Bilateral frontoparietal \\
\hline White matter abnormality & Yes & Yes \\
\hline Brainstem/cerebellum hypoplasia & Brainstem & Yes \\
\hline Corpus callosum abnormalities & No & $\begin{array}{l}\text { The corpus callosum without } \\
\text { rostrum }\end{array}$ \\
\hline
\end{tabular}

PMG: polymicrogyria, EEG: electroencephalography, M: male, F: female, Y: year

with BFPP consistently have such clinical findings as intellectual disability, epilepsy, ataxia, and dysconjugate gaze.

Herein we describe overlapping phenotypic features of BFPP syndrome without cerebellar hypoplasia in a Turkish patient with a homozygous nonsense mutation in the GPR56 gene.

\section{Material and Methods}

\section{Case reports}

The study protocol and the consent form were approved by the Dr. Behçet Uz Children's Hospital Ethics Committee. The parents of the three siblings were consanguineous (Fig 1A). Patient IV-1, IV-2, and IV-3 were aged 23 years, 25 years, and 14 years, respectively, now. The birth of each sibling was via uneventful spontaneous vaginal delivery, and auxological parameters were normal. The siblings, all with the same mutation in the GPR56 gene, had a similar clinical phenotype that included intractable epileptic seizures, intellectual disability and diparesis with profound spasticity. They need complete support in daily living. All affected siblings had focal onset bilateral tonic clonic seizure and were treated with anti-epileptic drugs, including valproate, levetiracetam, clonazepam, and lamotrigine. The age of onset of epileptic seizures of the affected siblings was 2-3 years. Though we did genetic analysis on the three siblings and their parents, we could only obtain MRI data for the index patient (Patient IV-3), who underwent further clinical and radiological investigations after identification of GPR56 mutation. On physical examination, patient IV-3 at age 14 years exhibited intellectual disability, and did not speak or communicate in any way. Fundus examination was normal and pupillary reflexes were intact. She could not follow moving objects. Furthermore, she had spastic diparesis. Deep-tendon reflexes were increased in both lower extremities. Also, she was unable to walk. Laboratory findings (complete blood count, liver, kidney, and thyroid function tests, CK (creatine kinase), uric acid, and vitamin B12 levels, and metabolic study) were normal. Abdominal ultrasonography (USG) was also normal. Long-term electroencephalogram (EEG) recordings showed rhythmic high amplitude bilateral frontal spike and waves with accompanying seizure which occurs every 


\begin{tabular}{|c|c|c|c|c|c|c|c|c|c|c|c|c|c|c|c|c|c|}
\hline 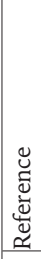 & 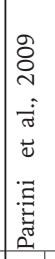 & 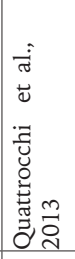 & 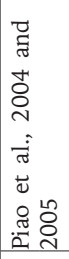 & 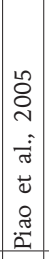 & 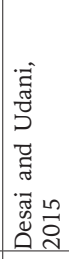 & 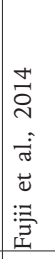 & 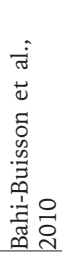 & 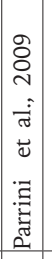 & 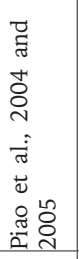 & 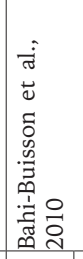 & 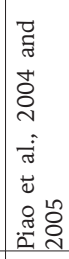 & 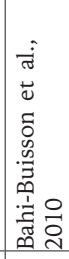 & 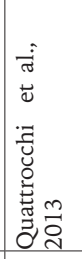 & 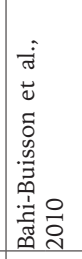 & 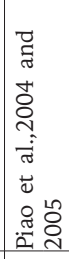 & 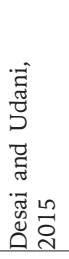 & 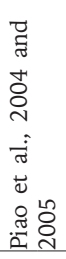 \\
\hline & & $\bar{\Xi}$ & & & & 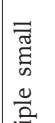 & $\begin{array}{l}0 \\
8 \\
0 \\
\vec{\sigma}\end{array}$ & & & & & & $\mathbb{\Xi}$ & & & & \\
\hline
\end{tabular}

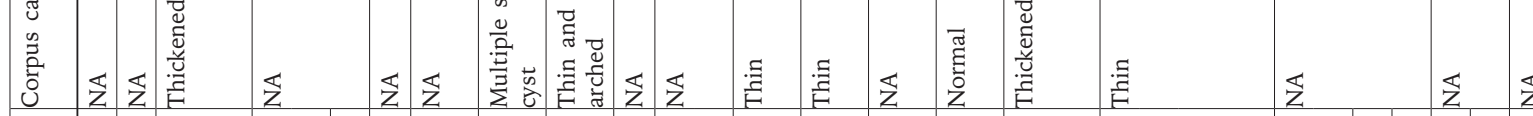

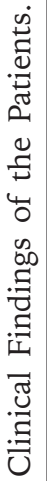

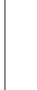

$\because \frac{5}{5}$

\section{空}

ธี

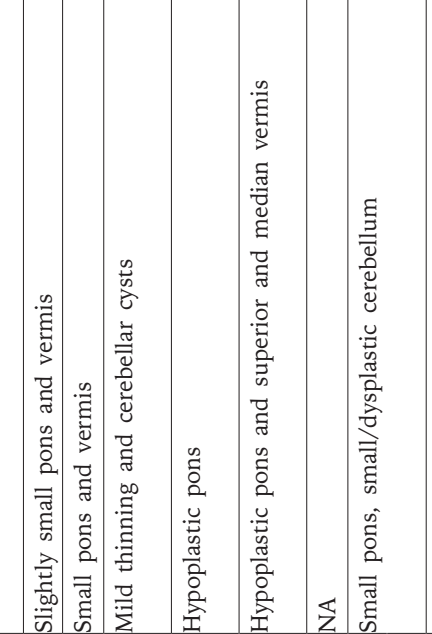

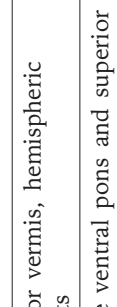

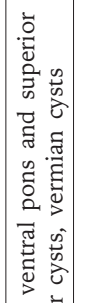

营

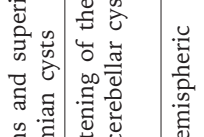

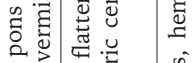

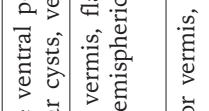

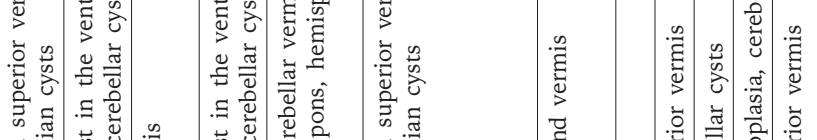

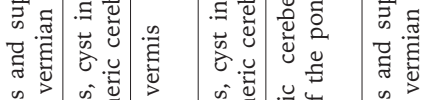

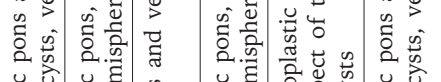

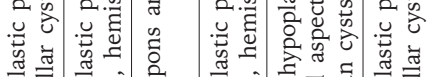

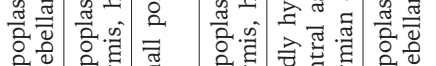

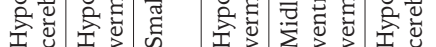

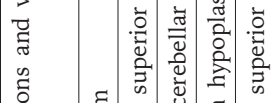

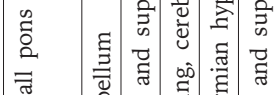

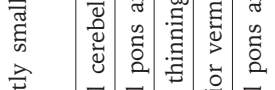

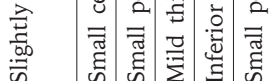

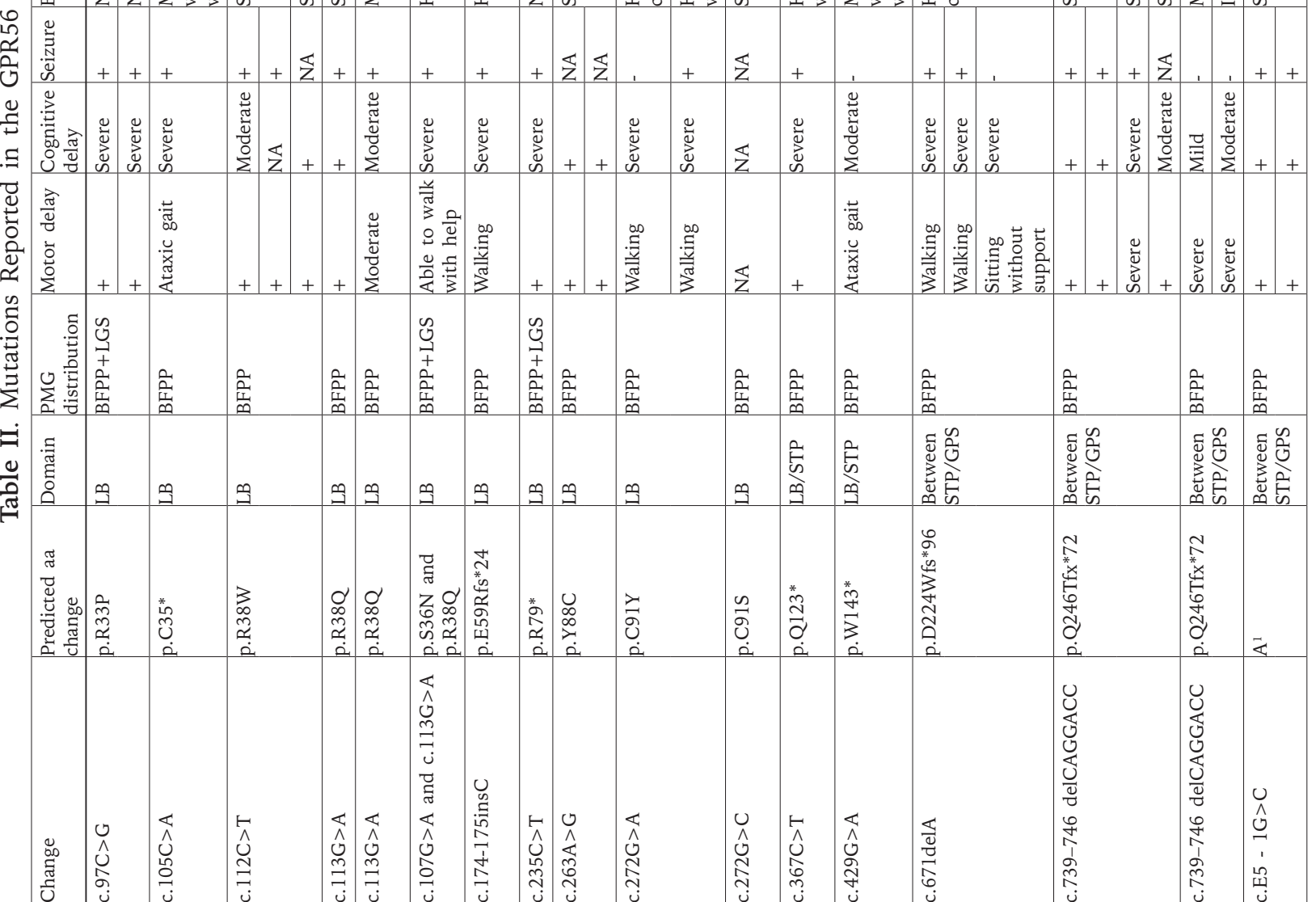




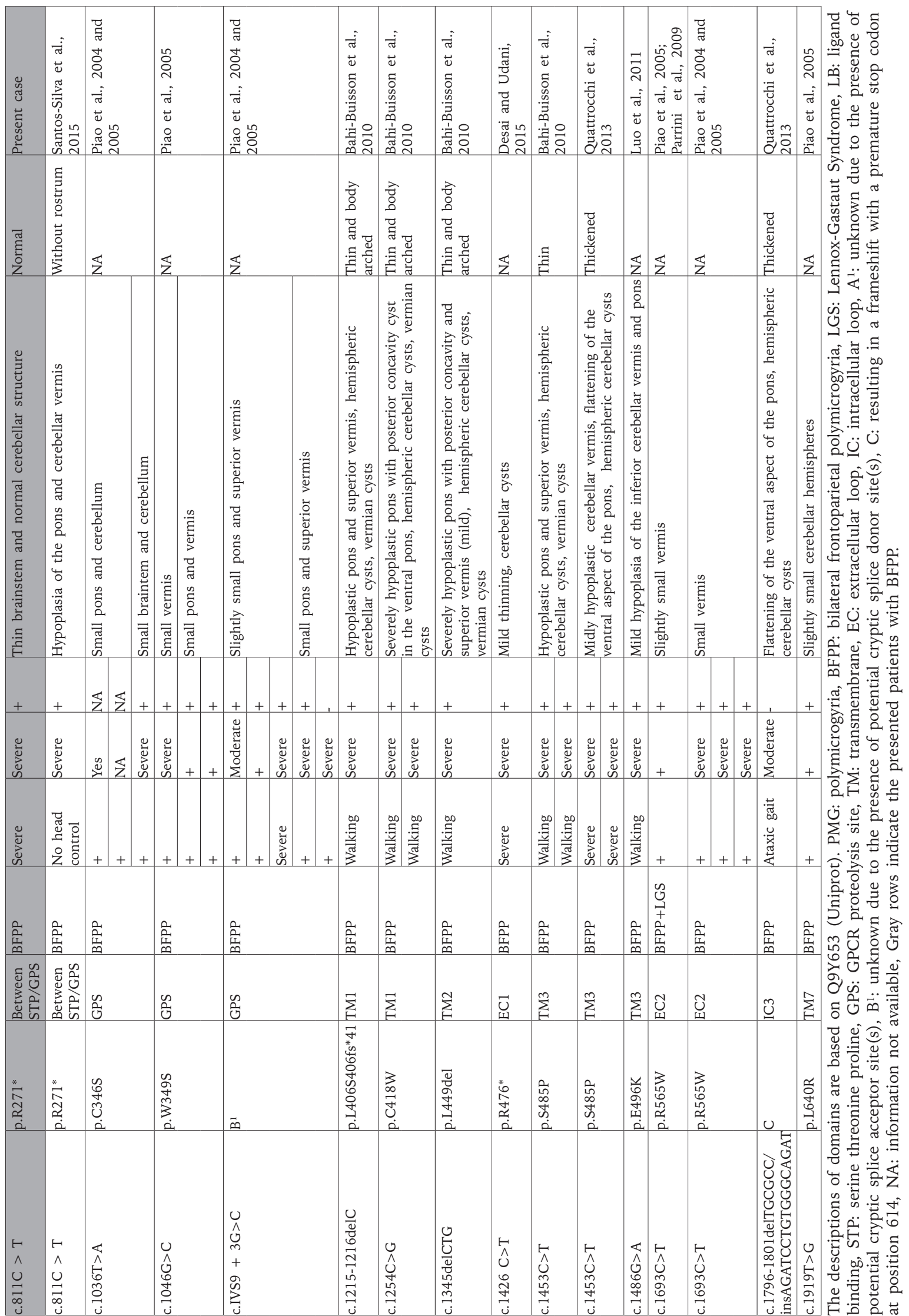




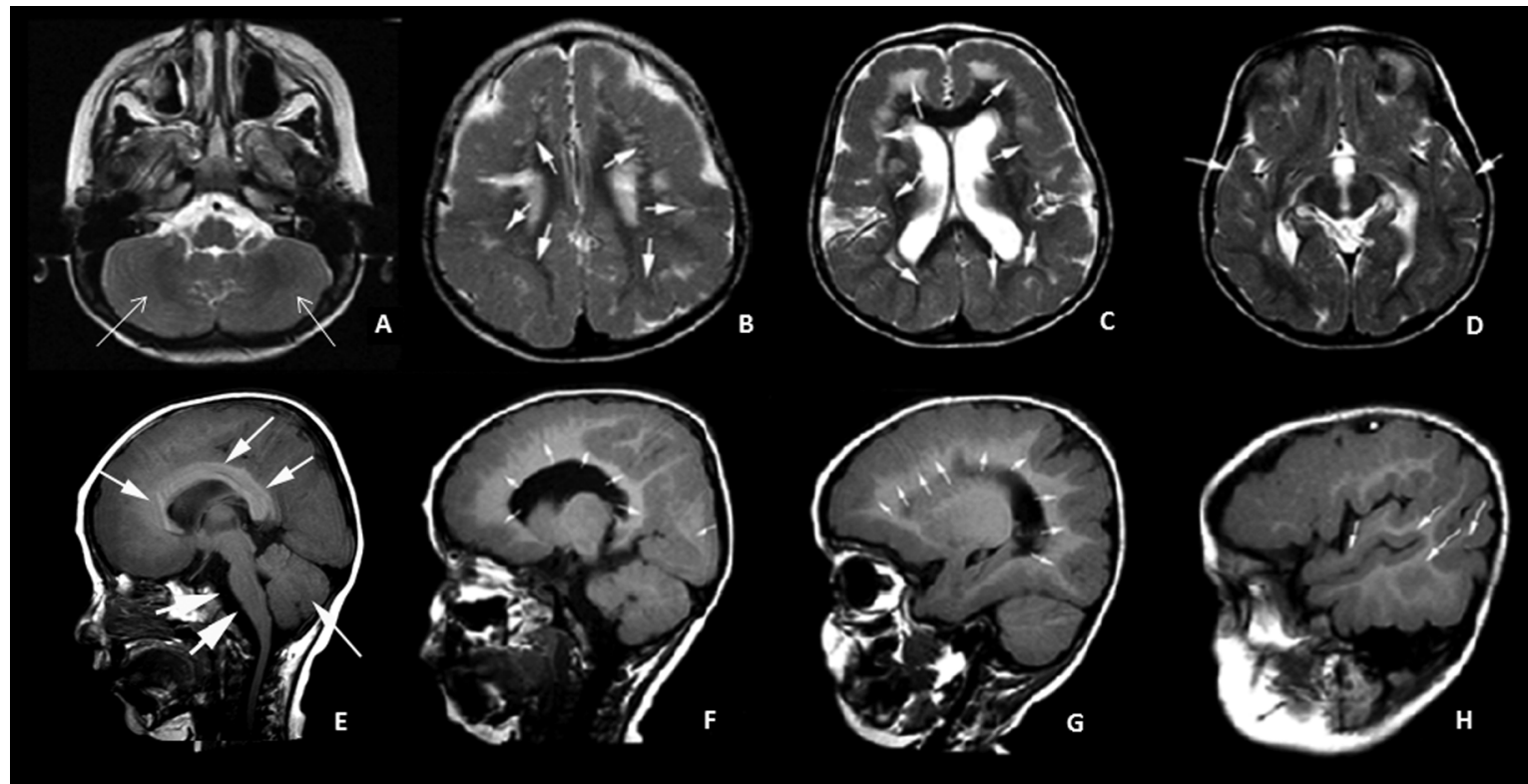

Fig 2. Cerebral and ponto-cerebellar MRI (Patient IV-3). Axial T2 weighted consecutive slices (arrows on B, C, D) and T1 weighted sagital slices (arrows on F, G, H) show distribution of polymicrogyria on frontal, parietal and occipital lobes. T1 weighted sagital slices shows the flattened pons and thin brainstem (thick arrows on E), the normal cerebellar structure (long arrow on E) and the normal corpus callosum (short arrows on E). Axial T2 weighted consecutive slice shows the normal cerebellar hemispheres (arrows on A). Temporal lobes are seen normal (arrows on F).

day, including bilateral tonic extensor spasms of the upper limbs, via video-EEG monitoring (Fig. S1). Bilateral prolonged visually evoked potential (VEP) latency was noted in the anterior visual pathways. Patient IV-3 showed the characteristic morphologic pattern of bilateral frontoparietal-occipital PMG (Fig. 2B, C, D, F, G and H) and brainstem hypoplasia (Fig $2 \mathrm{E})$ but did not show any cerebellar hypoplasia (Fig. 2A and E) in cranial MRI (Fig. 2). Also she does not have corpus callosum abnormalities (Fig. 2E). The phenotypic features of the three presented patients and the previously reported Portuguese patient that carried the same mutation are summarized in Table I.

\section{Genetic analysis}

Isolation of genomic DNA in peripheral blood samples obtained from the three patients, their parents and 100 controls was performed using the NucleoSpin ${ }^{\circledR}$ Blood L Kit, in accordance with the manufacturer's instructions (Machenery-Nagel, Germany), following provision of informed consent.

Whole exome sequencing (WES) and analysis pipeline

Fragmented (300-400 bp in length) DNA
(300-500 $\mu \mathrm{g})$ was used for library preparation using a KAPPA Library Preparation Kit (KAPPA Biosystems, MA, USA). Following quantification of the library via Qubit, 500 ng of library from each sample was used for the formation of pools. Exonic fragments from the pools were captured using SeqCap EZ v.3.0 (Roche, USA). Following amplification and quantitation using a KAPPA Library Quantification Kit (KAPPA Biosystems, US), the libraries were sequenced using TrueSeq Rapid Cluster and TruSeq SBS kits (Illumina Inc., USA), and a HiSeq 2500 system.

For each sample 7-11 GB of data were generated in read length of paired-ends (PE) $2 \times 104$. Illumina Real Time Analysis v.1.13 with standard parameters was used for real-time image analysis and base calling. hg19 was used for alignment of the PE reads using Burrows-Wheeler Alignment tool (BWA) ${ }^{8}$. Following alignment, SAMtools ${ }^{9}$ was used to remove polymerase chain reaction (PCR) duplicates, and the coverage rate for the targeted exonic regions was estimated using Browser Extensible Data (BED) tools. ${ }^{10}$ Local alignment was performed, and then SNP and INDEL calls with GATK11 and ANNOVAR ${ }^{12}$ were employed for functional annotation. After 


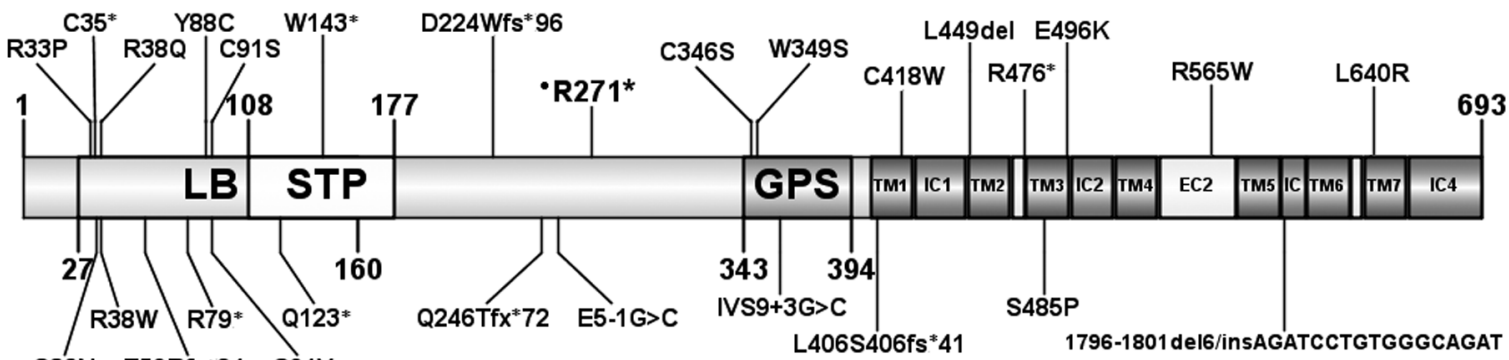

Fig 3. Schematic representation of the GPR56 protein showing mutations reported. LB: ligand binding; STP: serine threonine proline, GPS: GPCR proteolysis; TM: transmembrane; IC: intracellular loop; EC: extracellular loop; : represents the mutation detected in the presented family.

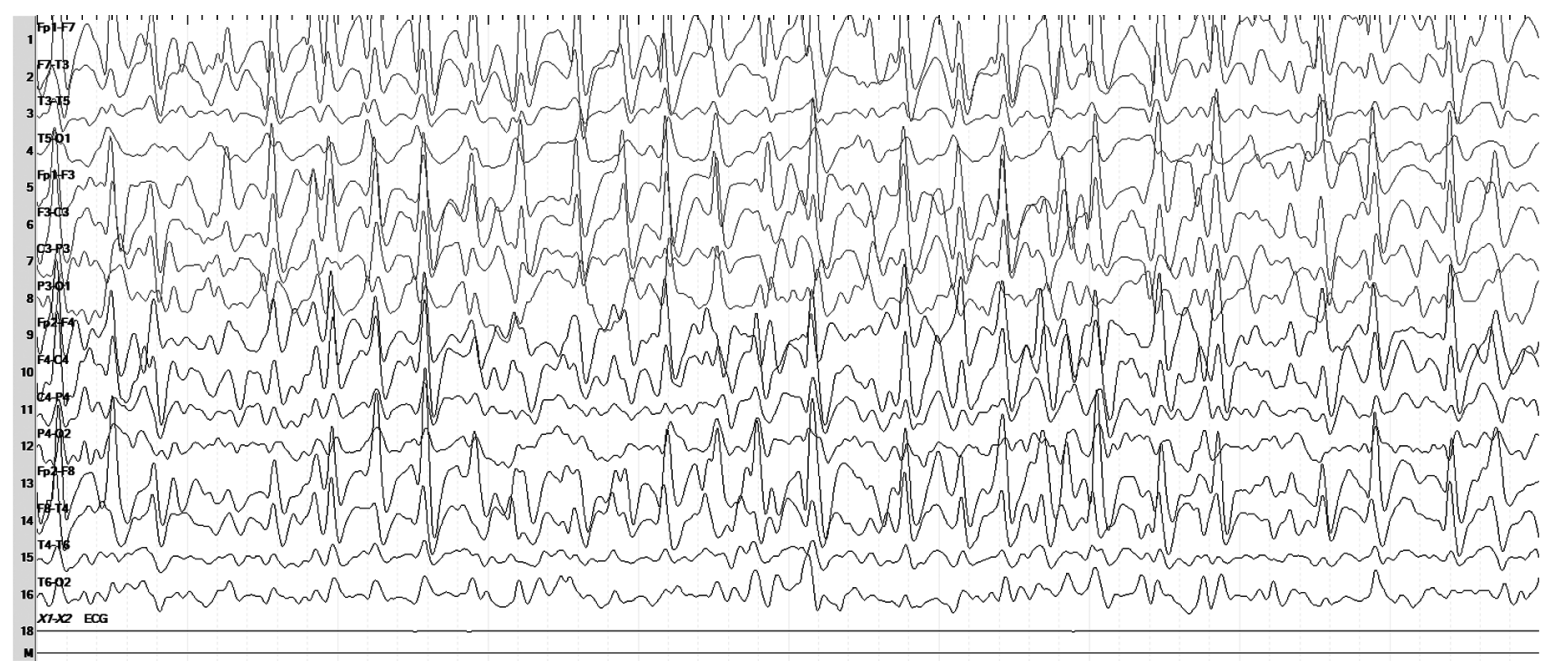

Fig S1. The interictal sleep EEG shows rhythmic high amplitude (150-160 $\mu$ V) bilateral frontal spike and waves on bilateral frontocentrotemporal regions (Patient IV-3).

the variants had been filtered, the diesease candidate variants were chosen according to the autosomal recessive segregation model, i.e. the parents (III-1 and III-2) were denoted as heterozygous and the 3 affected siblings (IV-1, IV-2 and IV-3) were denoted as homozygous variant. Following application of a segregation filter, 4 exonic-nonsynonymous variants in ACAN, GLTPD2, ASGR1 and ALOXE3 genes, and an exonic nonsense variant in GPR56 gene were investigated in greater detail as likely pathogenic variants. As variants in GPR56 had already been associated with malformation of cortical development (OMIM \#606854), the nonsense mutation in this gene was further investigated.

Validation and segregation analysis of the candidate variant via Sanger sequencing

The variation of interest identified via exome sequencing was confirmed via Sanger sequencing and specific restriction enzyme digestion of amplified PCR product. Exon 7 of GPR56 (accession number NM 005682, GRCh37/ hg19), including the variant, was amplified via PCR of genomic DNA using the upper primer 5'TTCCTGAAGAAATGGGCTTAG3' and the lower primer 5'ACAGGGAGATTTGCACATTG3'. Amplified PCR products were sequenced using an automated sequencer (ABI Genetic Analyzer 3730xi) with the lower primer. The PCR conditions employed are available upon request. According to the sequencing results, patients IV-1 and IV-2, and the parents were evaluated, but not patient IV-3. The quality of the Sanger chromatograms was poor for patient IV-3. So her variation was confirmed via PCR- restriction fragment length polymorphism (RFLP) analysis. Her amplified PCR product was digested using TaqI (New England Biolabs Inc., USA) restriction enzyme to identify the site of the mutation to be tested. The other 2 affected 
siblings and the parents were also included in this analysis. To exclude if this variant is a rare polymorphism, 100 Turkish controls (200 chromosomes) were screened by PCR-RFLP techniques. Also, it was screened in the Exome Aggregation Consortium (ExAC) database. It was analyzed the evolutionary conservation of arginine at position 271 using sequence alignment of this region in 13 different species.

\section{Results}

This study included three affected siblings and their consanguineous parents (Fig. 1A), and whole-exome sequencing in the three siblings showed a homozygous germline nonsense mutation (nomenclature according to HGVS: c. $811 \mathrm{C}>\mathrm{T}$, p.R271*) in GPR56 located in the seventh exon of the gene (Fig. 1B). The variant of interest, as identified via exome sequencing, was confirmed via Sanger sequencing and specific restriction enzyme digestion of amplified PCR product. According to the findings, the three siblings were homozygous and their parents were heterozygous for the mutation (Fig. 1B and C). The premature stop codon causes usually nonfunctional protein product. Several tests were used to predict the pathogenic effect of the p.R271* variant. Firstly, it was showed that there was no, in additional 100 controls (200 chromosomes), Turkish individual of the variant segregated with disease. According to this result it could be suggested that p.R271* variant may be disease causing mutation rather than a rare polymorphism. Secondly, the sequence alignment in various species showed that the arginine at position 271 was highly conserved and was predicted to negatively affect protein function (Fig. 1D). Thirdly, this variant was observed in only one allele in European population (Non-Finnish) among the available data of 120224 alleles in the Exome Aggregation Consortium (ExAC) database.

\section{Discussion}

Homozygous mutations in the GPR56 gene, which regulates migration of neural precursor cells, are associated with BFPP syndrome including white matter changes, brainstem and cerebellar involvement, and such clinical findings as intellectual disability, epilepsy and ataxia. ${ }^{4,5}$ The presented index patient partially exhibits the features of BFPP syndrome without cerebellar changes. Additionally, she had a homozygous GPR56 mutation and PMG was not limited to the bilateral frontoparietal regions, but also involved the occipital lobes. To date, 28 pathogenic GPR56 variations linked to the BFPP phenotype have been reported ${ }^{4-6,13-19}$ (Table II). Among these variants, p.R271* was detected in the three siblings in the present study. It has been previously reported in only one Portuguese patient. Although the Portuguese patient reported by Santos-Silva $\mathrm{R}$ et al. ${ }^{19}$ had the same mutation as presented in our patients, there are important clinical and radiological differences (Table I). The Portuguese patient had seizures provoked by hot water, macrocephaly, and a corpus callosum without rostrum. But, index patient did not show these phenotypic features. Despite of the phenotypic similarities in index patient and Portuguese patient, the potential causes of the variation in the expression of the p.R271* variant between the 2 affected families might be genetic or epigenetic factors beyond the GPR56 gene. Moreover, it can be difficult to detect subtle differences via MRI and CT, even for experienced radiologists.

The GPR56 gene is a member of the adhesion G-protein-coupled receptor (GPCR) family. The protein consists of three regions: 1 . a long extracellular $\mathrm{N}$-terminal region, including a signal peptide [SP], a serine threonine proline [STP] segment, and a ligand binding [LB] domain; 2. a GPCR proteolysis site (GPS) responsible for cleaving the protein into $\mathrm{N}$ - and C-terminal fragments; 3 . a seventransmembrane $\alpha$-helix (7TM) C-terminal region.6,20,21 The p.R271* mutation detected in the index patient and her siblings is located between the STP segment and GPS domain in the extracellular $\mathrm{N}$-terminal region of the protein which might predicted to cause a premature truncation with loss of the GPS domain and C-terminal region, along with three of the potential $7 \mathrm{~N}$-glycosylation sites of the GPR56 protein. Previously, five mutations have been reported between STP/GPS (Table II). Patients carrying three of them (D224Wfs*96, Q246Tfx*72 and W143*) and the presented patients are remarkable due to the presence of diffuse PMG. Earlier functional studies reported that the position of mutations in the GPR56 gene variably impair protein trafficking and 
cell surface expression21. Furthermore, BahiBuisson $\mathrm{N}$, et al. ${ }^{5}$ observed the effect of the position of mutation and type of substitution on clinical and radiological features. Luo $\mathrm{R}$ et al. ${ }^{15}$ suggested that E496K and R565W mutations in the $\mathrm{C}$-terminal region can have negative affect intracellular trafficking of GPR56 into the Golgi apparatus, but does not affect the proteolytic process of GPR56. In consideration of the findings of earlier studies shown in Table II and of the present study (Fig. 3), we suggest that mutations that lead to the absence of and changes to the $\mathrm{C}$-terminal region in GPR56 might be associated with our patients phenotypes. Nonetheless, the full extent of the biological consequences and potential role of the p.R271* mutation observed in the presented patients on the phenotype are not clear, and has yet to be experimentally verified by additional functional studies. These functional studies will provide insight into the mechanism of normal cortical development and regional patterning of the cerebral cortex as well as detecting the phenotypic spectrum of the mutation.

In conclusion, the present study reports the first consanguineous patient with BFPP syndrome without cerebellar hypoplasia, also having GPR56 gene mutation that was previously detected in only one Portuguese patient. The present findings show that the same mutation in GPR56 gene can have different phenotypic effects.

\section{Acknowledgements}

This study was supported by the Dokuz Eylul University Fund (2012.KB.SAG.026).

\section{REFERENCES}

1. Chang BS, Apse KA, Caraballo R, et al. A familial syndrome of unilateral polymicrogyria affecting the right hemisphere. Neurology 2006; 66: 133-135.

2. Jansen A, Andermann E. Genetics of the polymicrogyria syndromes. J Med Genet 2005; 42: 369-378.

3. Chang BS, Piao X, Bodell A, et al. Bilateral frontoparietal polymicrogyria: Clinical and radiological features in 10 families with linkage to chromosome 16. Ann Neurol 2003; 53: 596-606.

4. Piao X, Chang BS, Bodell A, et al. Genotype-phenotype analysis of human frontoparietal polymicrogyria syndromes. Ann Neurol 2005; 58: 680-687.

5. Bahi-Buisson N, Poirier K, Boddaert N, et al. GPR56related bilateral frontoparietal polymicrogyria: further evidence for an overlap with the cobblestone complex. Brain 2010; 133: 3194-3209.
6. Singer K, Luo R, Jeong S-J, Piao X. GPR56 and the developing cerebral cortex: cells, matrix, and neuronal migration. Mol Neurobiol 2013; 47: 186-196.

7. Bae BI, Tietjen I, Atabay KD, et al. Evolutionarily dynamic alternative splicing of GPR56 regulates regional cerebral cortical patterning. Science 2014; 343: 764 768.

8. Li H, Durbin R. Fast and accurate short read alignment with Burrows-Wheeler transform. Bioinformatics 2009; 25: $1754-1760$

9. Li H, Handsaker B, Wysoker A, et al. The Sequence Alignment/Map format and SAMtools. Bioinformatics 2009; 25: 2078-2079.

10. Quinlan AR, Hall IM. BEDTools: A flexible suite of utilities for comparing genomic features. Bioinformatics 2010; 26: 841-842.

11. DePristo MA, Banks E, Poplin R, et al. A framework for variation discovery and genotyping using nextgeneration DNA sequencing data. Nat Genet 2011; 43: 491-498.

12. Wang K, Li M, Hakonarson H. ANNOVAR: Functional annotation of genetic variants from high-throughput sequencing data. Nucleic Acids Res 2010; 38: e164.

13. Desai NA, Udani V. GPR56-Related Polymicrogyria: Clinicoradiologic profile of 4 patients. J. Child Neurol 2015; 30: 1819-1823.

14. Fujii Y, Ishikawa N, Kobayashi Y, Kobayashi M, Kato M. Compound heterozygosity in GPR56 with bilateral frontoparietal polymicrogria. Brain Dev 2014; 36: 528531.

15. Luo R, Yang HM, Jin Z, et al. A novel GPR56 mutation causes bilateral frontoparietal polymicrogyria. Pediatr. Neurol 2011; 45: 49-53.

16. Parrini E, Ferrari AR, Dorn T, Walsh CA, Guerrini R. Bilateral frontoparietal polymicrogyria, Lennox-Gastaut syndrome, and GPR56 gene mutations. Epilepsia 2009; 50: $1344-1353$.

17. Piao X, Hill RS, Bodell A, et al. G protein-coupled receptor-dependent development of human frontal cortex. Science 2004; 303: 2033-2036.

18. Quattrocchi CC, Zanni G, Napolitano A, et al Conventional magnetic resonance imaging and diffusion tensor imaging studies in children with novel GPR56 mutations: Further delineation of a cobblestone-like phenotype. Neurogenetics 2013; 14: 77-83.

19. Santos-Silva R, Passas A, Rocha C, et al. Bilateral frontoparietal polymicrogyria: A novel GPR56 mutation and an unusual phenotype. Neuropediatrics 2015; 46: 134-138.

20. Luo R, Jin Z, Deng Y, Strokes N, Piao X. Diseaseassociated mutations prevent GPR56-collagen III interaction. PLoS One 2012; 7: e29818.

21. Jin $\mathrm{Z}$, Tietjen $\mathrm{I}, \mathrm{Bu} \mathrm{L}$, et al. Disease-associated mutations affect GPR56 protein trafficking and cell surface expression. Hum Mol Genet 2007; 16: 1972 1985. 